\title{
THE BOUND STATES OF 3D Y-JUNCTION WAVEGUIDES
}

\author{
Pauliina Uusitalo \\ University of Oulu, Department of Electrical Engineering \\ P. O. Box 4500, 90014 Oulu, Finland; pauliina.uusitalo@oulu.fi
}

\begin{abstract}
The spectrum of the Dirichlet problem for Laplace operator is studied in three terminal tubes. The cross-section of the tubes is either a circle or a square. We show that these Y-shaped waveguides always have at least one eigenvalue in the discrete spectrum. In the regular case, that is, the angle between the cylinders is $\frac{2}{3} \pi$, there exists exactly one eigenvalue in the discrete spectrum. While the angle $2 \alpha$ between the arms is varying, we show that the number of the bound states remains to be one for $\alpha \in\left(\arctan \left(\frac{3}{4}\right), \frac{\pi}{2}\right]$ when the cross-section of the tubes is square. However, when the angle becomes sharp enough, the number of eigenvalues in the discrete spectrum increases. Moreover, it is shown that the eigenvalues are monotonously increasing when the angle $2 \alpha$ is in the interval $\left(0, \frac{2}{3} \pi\right)$ and are monotonously decreasing when $2 \alpha \in\left(\frac{2}{3} \pi, \pi\right]$.
\end{abstract}

\section{Physical background}

The development of nanotechnology has lead to smaller and smaller devices in electrical engineering. Over the last few decades there have been several both theoretical and applied studies to understand the physical behavior of such small nanometer sized waveguides.

The nanometer scale devices have attracted due to their excellent attributes. They have light weight, but they are still strong because of their elasticity. Moreover, they have exceptional electrical properties. These waveguides have either metallic or semi-conducting behavior depending on their geometrical structure.

These devices have a high purity and crystalline structure. Thus, the electron mean free path is greater than the diameter of the system. Therefore the scattering is unsubstantial and derives us to model the electron motion as a free particle in the infinite waveguide where the motion is limited inside the waveguide by posing the Dirichlet condition on the boundaries.

In this study we concentrate on the three-dimensional and three terminal nanotubes with either rectangular or circular cross-section. These waveguides are often called Y-junctions. Our intention is to investigate the existence of the non-trivial solutions of the Helmholtz equation:

$$
\begin{cases}-\Delta u(x)=\lambda u(x), & x \in \mathbf{Y}, \\ u(x)=0, & x \in \partial \mathbf{Y} .\end{cases}
$$

In particular, those solutions which have the finite energy and the eigenvalue corresponding to such an eigenfunction is located below the continuous spectrum. The continuous spectrum is where the electron propagation can occur as the contrary to the discrete spectrum where the eigenfunction is localized.

doi:10.5186/aasfm.2015.4023

2010 Mathematics Subject Classification: Primary 35Q40, 35J05, 58C40, 81Q10.

Key words: Quantum waveguide, spectrum, bound state. 
The first theoretical explorations of the existence of the bound states were made more than quarter of a century ago. The appearance of the bound states in twisting and curved waveguides in two and three dimensions were illustrated in the papers $[6,7]$ and $[8]$.

The Y-branch waveguide has some technical applications as a miniaturized switch, where the electrons are directed to one of the two branches (see, e.g., [2]). Also it has been presupposed in [16] that Y-branches could be connected to each other to form logic circuits.

Related to the waveguide at issue, the regular two-dimensional Y-junction waveguide was studied in [15]. The width of one arm was varying while the other two were set to have unit width. The discrete spectrum was discovered to be empty when the width of the arm exceeded 1.25.

\section{Introduction}

Let $\omega \in \mathbf{R}^{2}$ be a domain with a Lipschitz boundary $\partial \omega$ and $\Pi^{H}$ a cylinder which is bounded from below, that is, $\Pi^{H}=\left\{x=(y, z) \in \mathbf{R}^{2} \times \mathbf{R}: y \in \omega,-H(y)<z\right\}$, where $H$ is a Lipschitz continuous profile function on $\bar{\omega}$. We will consider the domain $\mathbf{Y}$, where union of the three cylinders forms a waveguide of shape of letter "Y".

We focus on examine the waveguides in two different cases. The rectangular case is when the cross-section is square, that is, $\omega_{R}=\left\{y=\left(y_{1}, y_{2}\right):-\frac{1}{2}<y_{1}, y_{2}<\frac{1}{2}\right\}$. The profile function $H$ is continuous piecewise linear function which form the cylinder to have pointed end (see the left side of Fig. 1). We denote the rectangular waveguide by $\mathbf{Y}_{R}$.
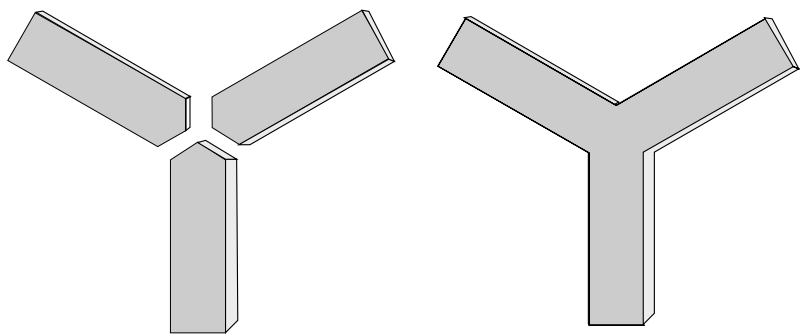

Figure 1. The pointed cylinders and the branching waveguide with rectangular cross-section.

While the cross-section is a circle, $\omega_{C}=\left\{y=\left(y_{1}, y_{2}\right):|y|<\frac{1}{2}\right\}$, that is the circle is of radius $\frac{1}{2}$. Again, the cylinders are bounded by the piecewise linear profile functions. For the circular case, we use the notation $\mathbf{Y}_{C}$ (see Fig. 4 and 6).

In Sections 3 and 4 we focus attention on the regular case, where the angle between the cylinders is $\frac{2 \pi}{3}$ while in Section 5 we investigate the spectrum when the angle $\alpha$ is varying. Throughout the paper, we use notation $\mathbf{Y}_{R}$ or $\mathbf{Y}_{C}$ without a superscript for the regular cases and with the superscript $\alpha$ the cases where the angle is varying.

We study the spectral properties of the Dirichlet-Laplacian (1) in the branching waveguide $\mathbf{Y}$. This can be rewritten in the variational form

$$
(\nabla u, \nabla v)_{\mathbf{Y}}=\lambda(u, v)_{\mathbf{Y}}
$$

That is, we need to find an eigenpair $(\lambda, u)$, where $\lambda \in \mathbf{C}$ is a spectral parameter and a non-trivial $u \in H_{0}^{1}(\mathbf{Y})$ is an eigenfunction. The function $u$ satisfies the integral identity for any compactly supported smooth test function $v \in C_{0}^{\infty}(\mathbf{Y})$ vanishing at the boundary $\partial \mathbf{Y}$. The natural scalar product in Lebesgue space $L^{2}(\mathbf{Y})$ is denoted 
by $(\cdot, \cdot)_{\mathbf{Y}}$. Moreover, $H_{0}^{1}(\mathbf{Y})$ is a subspace of functions in Sobolev space $H^{1}(\mathbf{Y})$ that vanish on the boundary. Since the bi-linear form on the left in $(2)$ is closed in $H_{0}^{1}(\mathbf{Y})$ and positive definite, the formulation gives rise to an unbounded positive definite and self-adjoint operator $A$ in Hilbert space $H_{0}^{1}$ (Y) (see, e.g., [3, Ch. 10]).

The spectrum of the operator $A$ is located on the positive semi-axis $\mathbf{R}_{+}$. Moreover, the operator $A$ has a continuous spectrum $\sigma^{c}=\left[\lambda_{\dagger},+\infty\right)$, where $\lambda_{\dagger}$ is the first eigenvalue of the problem on the cross-section $\omega:(\nabla u, \nabla v)_{\omega}=\lambda(u, v)_{\omega}$. The discrete spectrum $\sigma^{d}$ lies in $\left(0, \lambda_{\dagger}\right)$, if it exists.

\section{The rectangular cross-section}

Let us now examine the waveguide with the rectangular cross-section. First, the threshold $\lambda_{1}$ for the continuous spectrum can be found by solving the following problem

$$
\begin{cases}-\Delta u=\lambda u, & y \in \omega_{R} \\ u=0, & y \in \partial \omega_{R} .\end{cases}
$$

That is, the first eigenvalue is $\lambda_{1}=2 \pi^{2}$ and hence $\sigma^{c}\left(\mathbf{Y}_{R}\right)=\left[2 \pi^{2},+\infty\right)$.

We are interested to know if there exists any eigenvalue below the continuous spectrum, namely, in the discrete spectrum $\sigma^{d}\left(\mathbf{Y}_{R}\right)$.

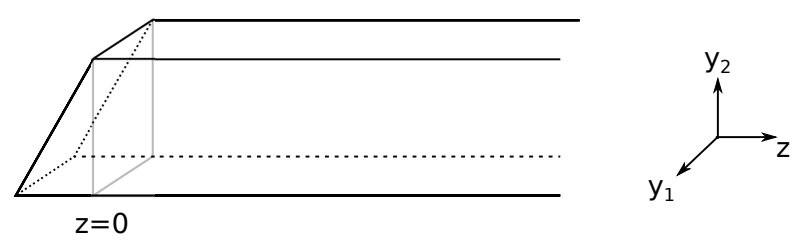

Figure 2. The semi-cylinder of $\mathbf{V}$.

Lemma 1. The discrete spectrum $\sigma^{d}\left(\mathbf{Y}_{R}\right)$ is non-empty.

Proof. Let us first consider the V-shaped waveguide, $\mathbf{V}$, where two cylinders with slanted ends are joint together so that the angle between them is $\frac{2 \pi}{3}$ (see Fig. 2) and make an assumption that the lower bound $\sigma_{\mathbf{V}}$ of the spectrum is $2 \pi^{2}$, i.e., the discrete spectrum is empty. By [3, Ch. 10.1, 10.2], the lower bound is found by

$$
\underline{\sigma_{\mathbf{V}}}=\inf _{u \in H_{0}^{1}(\mathbf{V})} \frac{\left\|\nabla u ; L^{2}(\mathbf{V})\right\|^{2}}{\left\|u ; L^{2}(\mathbf{V})\right\|^{2}} .
$$

Due to the symmetry of $\mathbf{V}$, it is enough to consider one side of the waveguide. We use the local Cartesian coordinate system and define a test function

$$
u^{\varepsilon}(y, z)=v^{\varepsilon}(y, z)+\sqrt{\varepsilon} w(y, z),
$$

where $w(y, z) \in H_{0}^{1}(\mathbf{V})$ is a compactly supported smooth function and

$$
v^{\varepsilon}(y, z)= \begin{cases}\cos \left(\pi y_{1}\right) \cos \left(\pi y_{2}\right), & z<0, \\ \cos \left(\pi y_{1}\right) \cos \left(\pi y_{2}\right) e^{-\varepsilon z}, & z \geq 0,\end{cases}
$$

where $\varepsilon>0$. The function $u^{\varepsilon}$ is continuous, but the normal derivative is

$$
\partial_{\nu} u^{\varepsilon}= \begin{cases}0, & z<0 \\ \varepsilon \cos \left(\pi y_{1}\right) \cos \left(\pi y_{2}\right), & z \geq 0\end{cases}
$$


that is, to have the test function $u^{\varepsilon}$ in $H_{0}^{1}(\mathbf{V})$, we choose the function $w$ so that its support is located in the neighborhood of the slanted end, denoted by $\Xi$. Now,

$$
\begin{aligned}
& \left\|\nabla u^{\varepsilon} ; L^{2}(\mathbf{V})\right\|^{2}-2 \pi^{2}\left\|u^{\varepsilon} ; L^{2}(\mathbf{V})\right\|^{2}=\left\|\nabla v ; L^{2}(\mathbf{V})\right\|^{2}-2 \pi^{2}\left\|v ; L^{2}(\mathbf{V})\right\|^{2} \\
& +2 \sqrt{\varepsilon} \int_{\mathbf{V}}\left(\nabla v \nabla w-2 \pi^{2} v w\right) \mathrm{d} x+\varepsilon\left(\left\|\nabla w ; L^{2}(\mathbf{V})\right\|^{2}-2 \pi^{2}\left\|w ; L^{2}(\mathbf{V})\right\|^{2}\right),
\end{aligned}
$$

where the first two terms on right equals $\frac{1}{4} \varepsilon$ and the last term is $c \varepsilon>0$ due to the assumption of a lower bound. We apply Green's formula and write (4) into a form

$$
2 \sqrt{\varepsilon} \int_{\mathbf{V}}\left(\nabla v \nabla w-2 \pi^{2} v w\right) \mathrm{d} x+C \varepsilon=2 \sqrt{\varepsilon} \int_{\partial \Xi} w \partial_{\nu} v \mathrm{~d} s+C \varepsilon
$$

where $w$ can be selected so that the last integral on right becomes negative. Hence, the value of (4) is less than $-\sqrt{\varepsilon}+C \varepsilon$. For the small values of $\varepsilon>0$ this contradicts with the assumption since

$$
\underline{\sigma_{\mathbf{V}}}=\inf _{u \in H_{0}^{1}(\mathbf{V})} \frac{\left\|\nabla u ; L^{2}(\mathbf{V})\right\|^{2}}{\left\|u ; L^{2}(\mathbf{V})\right\|^{2}} \leq \frac{\left\|\nabla u^{\varepsilon} ; L^{2}(\mathbf{V})\right\|^{2}}{\left\|u^{\varepsilon} ; L^{2}(\mathbf{V})\right\|^{2}}<2 \pi^{2} .
$$

Thus, the discrete spectrum $\sigma^{d}(\mathbf{V})$ of the operator $A$ related to the problem (1) in the $\mathrm{V}$-shaped waveguide is non-empty. Therefore there is at least one eigenvalue $\lambda_{\mathbf{V}}$ with the corresponding eigenfunction $u_{\mathbf{V}}$. We extend the eigenfunction as zero from $\mathbf{V}$ onto $\mathbf{Y}_{R}$. Hence the lower bound is

$$
\underline{\sigma_{\mathbf{Y}_{R}}}=\inf _{u \in H_{0}^{1}\left(\mathbf{Y}_{R}\right)} \frac{\left\|\nabla u ; L^{2}\left(\mathbf{Y}_{R}\right)\right\|^{2}}{\left\|u ; L^{2}\left(\mathbf{Y}_{R}\right)\right\|^{2}} \leq \frac{\left\|\nabla u_{\mathbf{V}} ; L^{2}\left(\mathbf{Y}_{R}\right)\right\|^{2}}{\left\|u_{\mathbf{V}} ; L^{2}\left(\mathbf{Y}_{R}\right)\right\|^{2}}<2 \pi^{2},
$$

which guarantees the non-emptiness of the discrete spectrum.

Lemma 2. The multiplicity of the discrete spectrum $\sigma^{d}\left(\mathbf{Y}_{R}\right)$ equals one.

Proof. We assume that at least two eigenvalues appear in the discrete spectrum and show that such assumption leads to a contradiction. By the max-min principle (see $[3, \S 10.2 .2]$ ), the $n$th eigenvalue can be represented by

$$
\lambda_{n}=\max _{\mathcal{E}_{n}} \inf _{u \in \mathcal{E}_{n} \backslash\{0\}} \frac{\left\|\nabla u ; L^{2}(\mathbf{Y})\right\|^{2}}{\left\|u ; L^{2}(\mathbf{Y})\right\|^{2}},
$$

where $\mathcal{E}_{n}$ is a subspace of $H_{0}^{1}(\mathbf{Y})$ of co-dimension $n-1$.

We split the waveguide $\mathbf{Y}_{R}$ into three semi-cylinders $\Pi_{R}$ with the right-angled ends. In the middle there is left a prism $P$, which has an equilateral triangle $T$ as a base (see Fig. 3).

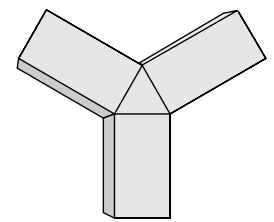

Figure 3. The division of the $\mathbf{Y}_{R}$. 
We consider the mixed boundary value problem on the prism $P$ as follows

$$
\begin{cases}-\Delta v=\mu v, & x \in P \\ \partial_{\nu} v=0, & \left(y_{2}, z\right) \in \partial T,-\frac{1}{2}<y_{1}<\frac{1}{2} \\ v=0, & \left(y_{2}, z\right) \in T, y_{1}= \pm \frac{1}{2}\end{cases}
$$

The first eigenvalue of Neumann-Laplacian is $\mu_{1}^{T}=0$ with the corresponding eigenfunction $v_{1}^{T}$, which is a constant. By [9], the second and third eigenvalues are $\mu_{2}^{T}=\mu_{3}^{T}=\frac{16}{9} \pi^{2}$. By the separation of variables, that is, in view of the third dimension, the first eigenvalue of the problem (9) on the prism is $\mu_{1}=\pi^{2}$ with the corresponding eigenfunction $v_{1}=c \cos \left(\pi y_{1}\right)$, where $c \in \mathbf{R}$. The second eigenvalue $\mu_{2}^{P}=\frac{25}{9} \pi^{2}$.

We set

$$
\mathcal{E}_{2}^{\perp}\left(\mathbf{Y}_{R}\right)=\left\{u \in H_{0}^{1}\left(\mathbf{Y}_{R}\right): \int_{P} u(x) v_{1}(x) \mathrm{d} y \mathrm{~d} z=0\right\}
$$

where $v_{1}(x)$ is the first eigenfunction of the problem (9). For any $u \in \mathcal{E}_{2}^{\perp}$, the inequality

$$
\frac{\left\|\nabla u ; L^{2}(P)\right\|^{2}}{\left\|u ; L^{2}(P)\right\|^{2}}>2 \pi^{2}
$$

is vindicated since $\mu_{2}^{P}$ is greater than the threshold $2 \pi^{2}$.

We employ the Friedrichs inequality for the cylinders $\Pi_{R}$, that is,

$$
\begin{aligned}
& 2 \pi^{2} \int_{-\frac{1}{2}}^{\frac{1}{2}} \int_{-\frac{1}{2}}^{\frac{1}{2}}\left|u\left(y_{1}, y_{2}, z\right)\right|^{2} \mathrm{~d} y \\
& \leq \int_{-\frac{1}{2}}^{\frac{1}{2}} \int_{-\frac{1}{2}}^{\frac{1}{2}}\left(\left|\frac{\partial}{\partial y_{1}} u\left(y_{1}, y_{2}, z\right)\right|^{2}+\left|\frac{\partial}{\partial y_{2}} u\left(y_{1}, y_{2}, z\right)\right|^{2}\right) \mathrm{d} y .
\end{aligned}
$$

Integrating over the variable $z \in(0, \infty)$, we obtain

$$
2 \pi^{2}\left\|u ; L^{2}\left(\Pi_{R}\right)\right\|^{2} \leq\left\|\nabla u ; L^{2}\left(\Pi_{R}\right)\right\|^{2} .
$$

Let us presume that $\lambda_{2}$ belongs to the discrete spectrum, that is, $\lambda_{2} \in\left(\lambda_{1}, 2 \pi^{2}\right)$. Due to the assumption, we have

$$
2 \pi^{2}>\lambda_{2} \geq \inf _{u \in \mathcal{E}_{2}^{\perp} \backslash\{0\}} \frac{\left\|\nabla u ; L^{2}\left(\mathbf{Y}_{R}\right)\right\|^{2}}{\left\|u ; L^{2}\left(\mathbf{Y}_{R}\right)\right\|^{2}} .
$$

The sum of inequalities (10) and (12) leads to

$$
\frac{\left\|\nabla u ; L^{2}\left(\mathbf{Y}_{R}\right)\right\|^{2}}{\left\|u ; L^{2}\left(\mathbf{Y}_{R}\right)\right\|^{2}} \geq 2 \pi^{2}, \quad \text { for } u \in \mathcal{E}_{2}^{\perp}\left(\mathbf{Y}_{R}\right)
$$

This contradicts with (13), and therefore the lemma is verified. 


\section{The circular cross-section}

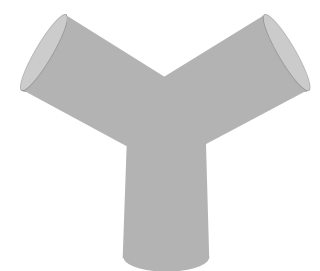

Figure 4. The branching waveguide with circular cross-section.

We now turn into the problem with the circular cross-section (see Fig. 4). To find the threshold of the continuous spectrum $\lambda_{\dagger}^{C}$ we solve the Dirichlet problem for the Laplacian on the cross-section $\omega_{C}:(\nabla u, \nabla v)_{\omega_{C}}=\lambda(u, v)_{\omega_{C}}$. We change over to the polar coordinates to see that the first eigenvalue is $\lambda_{1}=4 j_{0,1}^{2}$, where $j_{0,1} \approx 2.4048$ is the first positive root of Bessel function of order 0 (see, e.g., [5]). Thus, the continuous spectrum is $\sigma^{c}\left(\mathbf{Y}_{C}\right)=\left[4 j_{0,1}^{2},+\infty\right)$.

The existence of the eigenvalues in the discrete spectrum is studied in several types of waveguides. In the paper [1] it was shown with the help of the comparison principle that for any planar and broken waveguide there exists at least one eigenvalue in the discrete spectrum (see also [7]). In the paper [6], the non-emptiness of the discrete spectrum was verified for two and three dimensional bend tubes with equidistant width. Moreover, the result was extended to any number of dimensions in [8]. Applying to these results, we take a curved tube which is contained in the cranked V-shaped waveguide (see Fig. 5). Applying the results of [6] it is known that there exists at least one bound state. By the comparison principle, the existence of bound state in the discrete spectrum $\sigma_{C}^{d}$ is proven.

Moreover, in the paper [13], it was proven with the support of the variational method that whenever the profile function $H(y)$ of the cylinder is continuous and piecewise linear, the discrete spectrum in the case of the branching waveguide can not be empty. In fact, the proof given to the rectangular case in Section 3 is an example of it. (See also [4], where thin cylinders with distorted ends are studied.) Thus to derive corresponding lemma as in the rectangular case in Lemma 2, it remains to show the following lemma.

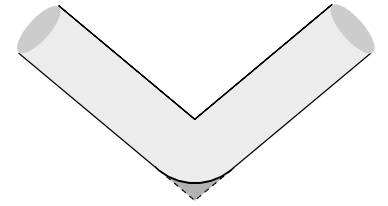

Figure 5. The curved waveguide.

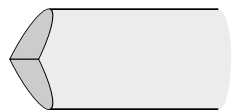

Figure 6. The branch of $\mathbf{Y}_{C}$.

Lemma 3. The discrete spectrum of $\sigma^{d}\left(\mathbf{Y}_{C}\right)$ contains exactly one eigenvalue.

Proof. We divide the waveguide into three identical cylinders with straight ends. An element left in the middle, $\tilde{P}$, is a conical type part, which resembles the prism $P$. Instead of this element $\tilde{P}$, we consider the prism $P$ with the mixed boundary problem given in (9). Since $\tilde{P} \subset P$, the eigenvalues $\mu_{n}^{\tilde{P}} \geq \mu_{n}^{P}$ by the comparison principle. Therefore, $\mu_{1}^{\tilde{P}} \geq \pi^{2}$ and $\mu_{2}^{\tilde{P}} \geq \frac{25}{9} \pi^{2}>4 j_{0,1}^{2}$. Thus, we employ the inequality

$$
\frac{\left\|\nabla u ; L^{2}(\tilde{P})\right\|^{2}}{\left\|u ; L^{2}(\tilde{P})\right\|^{2}}>4 j_{0,1}^{2},
$$


where a function $u \in H_{0}^{1}\left(\mathbf{Y}_{C}\right)$ satisfies the orthogonality condition $\int_{\tilde{P}} u(x) v_{1}(x) \mathrm{d} y \mathrm{~d} z$ $=0$, where $v_{1}$ is the corresponding eigenfunction to $\mu_{1}^{\tilde{P}}$.

Integrating over the variable $z \in(0, \infty)$ in the Friedrichs inequality, we obtain

$$
4 j_{0,1}^{2}\left\|u ; L^{2}\left(\Pi_{C}\right)\right\|^{2} \leq\left\|\nabla u ; L^{2}\left(\Pi_{C}\right)\right\|^{2} .
$$

Summing up the above inequalities, we obtain

$$
\frac{\left\|\nabla u ; L^{2}\left(\mathbf{Y}_{C}\right)\right\|^{2}}{\left\|u ; L^{2}\left(\mathbf{Y}_{C}\right)\right\|^{2}} \geq 4 j_{0,1}^{2}, \text { for } u \in \mathcal{E}_{2}^{\perp}\left(\mathbf{Y}_{C}\right)
$$

where $u$ satisfies one orthogonality condition. Now the the assumption $\lambda_{2} \in \sigma^{d}\left(\mathbf{Y}_{C}\right)$ and the max-min principle (8) contradict.

\section{The discrete spectrum when the angle is varying}

Let now the angles vary between the cylinders. We set the angle between the arms to be $2 \alpha$, where $\alpha \in\left(0, \frac{\pi}{2}\right]$. Then the other two angles are $\pi-\alpha$. We denote the waveguide by $\mathbf{Y}^{\alpha}$, where the angles are varying. At first, we concentrate purely on the rectangular cross-section. The same reasoning as in the earlier section ensures the non-emptiness of the discrete spectrum $\sigma^{d}\left(\mathbf{Y}_{R}^{\alpha}\right)$.

For example, in [1] and [14] the two-dimensional broken waveguides were studied with the varying angle. Altogether it was noticed that the multiplicity of the discrete spectrum increases infinitely as the angle approaches to zero. Again, in the paper [6] it was shown that when the tube is slightly bent, only one bound state appears. For example, for the two-dimensional V-shaped waveguide it was shown in [14] that the multiplicity of the discrete spectrum is exactly one whenever $\alpha_{V} \in\left[\arctan \left(\sqrt{\frac{2}{5}}\right), \frac{\pi}{2}\right)$. Due to the lack of the domain monotonicity, the same may not hold for $\mathbf{Y}_{R}^{\alpha}$. However, similar result is shown for $\mathbf{Y}_{R}^{\alpha}$ when $\alpha \in\left[\arctan \left(\frac{3}{4}\right), \frac{\pi}{2}\right]$, see Lemma 6 and Lemma 7.

To find the angles when only one eigenvalue lies in the discrete spectrum, we divide $\mathbf{Y}_{R}^{\alpha}$ in the following way. In the middle, we set a cuboid $Q$ of width and length 1 and height $a=\frac{2-\cos (\alpha)}{2 \sin (\alpha)}$. In the arms there exist two right angled prisms. The prisms $P_{1}$ and $P_{2}$, have a right-angled triangle $T_{1}$ and $T_{2}$, respectively, as a cross-section. For the bigger triangle $T_{1}$ one leg is $b=\frac{2-\cos (\alpha)}{2}$ and the hypotenuse $a$. In the smaller triangle $T_{2}$, one leg is $1-b$ and the hypotenuse $\frac{1}{2}$, see Fig. 7 .

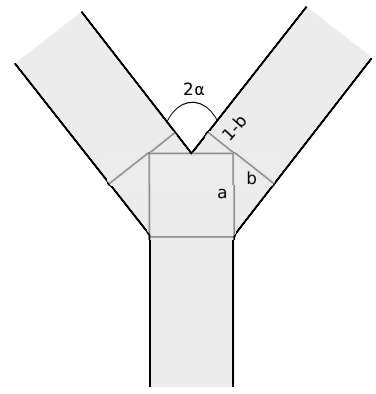

Figure 7. The division of the branching waveguide with the varying angle $\alpha$.

We pose Dirichlet boundary conditions on the sides of the $Q$, where $y_{1}= \pm \frac{1}{2}$. On the other sides we pose Neumann boundary condition. The first eigenvalue $\mu_{1}=\pi^{2}$ 
and the second $\mu_{2}=\pi^{2}+\min \left\{\pi^{2}, \frac{\pi^{2}}{a^{2}}\right\}$. Thus, the request to have the second eigenvalue greater than the threshold, we obtain that the height $a$ must be less or equal to 1 . Therefore the possible values of $\alpha$ are restricted to the interval:

$$
\alpha \in\left[\arctan \left(\frac{3}{4}\right), \frac{\pi}{2}\right] \text {. }
$$

For the prisms $P_{1}$ and $P_{2}$, we first consider the eigenvalue problems in the triangles $T_{1}$ and $T_{2}$. To the smaller triangle, having Neumann boundary condition on the hypotenuse and on one leg and Dirichlet condition on the other leg, the first eigenvalue is estimated by Friedrichs inequality (see for example [13]) to be $\mu_{1}\left(T_{2}\right) \geq \frac{\pi^{2}}{4(1-b)^{2}}$. Since $(1-b)^{2} \leq \frac{1}{4}$, the first eigenvalue $\mu_{1}\left(T_{2}\right)>\pi^{2}$ for every $\alpha \in\left[0, \frac{\pi}{2}\right)$. Thus, taking into account the third coordinate $y_{1}$, the first eigenvalue in the prism $P_{2}$ is $\mu_{1}\left(P_{2}\right)>2 \pi^{2}$. We set $\alpha=\frac{\pi}{4}$ and show the following.

Lemma 4. The discrete spectrum of $\mathbf{Y}_{R}^{\frac{\pi}{4}}$ contains exactly one eigenvalue.

Proof. The triangles $T_{1}$ and $T_{2}$ are now isosceles right triangles. On the isosceles right triangles the eigenvalue problem can be solved by separation variables (see, e.g., [11]). We first view the eigenvalues in the square $S$ with side length 1 . Dirichlet boundary conditions is posed on the sides $x_{1}=0$ and $x_{2}=0$ and Neumann condition on the other two sides. The eigenvalues are then $\mu(S)=\frac{\pi^{2}}{4}\left((2 m+1)^{2}+(2 n+1)^{2}\right)$ and the eigenfunctions $u_{S}(x)=\sin \left(\frac{2 m+1}{2} \pi x_{1}\right) \sin \left(\frac{2 n+1}{2} \pi x_{2}\right)$.

The combination of the eigenfunctions $u_{S}(x)$ solves the Laplace problem with mixed boundary conditions on the isosceles triangle, that is,

$$
u_{T}(x)=\sin \left(\frac{2 m+1}{2} \pi x_{1}\right) \sin \left(\frac{2 n+1}{2} \pi x_{2}\right)+\sin \left(\frac{2 n+1}{2} \pi x_{2}\right) \sin \left(\frac{2 m+1}{2} \pi x_{1}\right) .
$$

The eigenvalues are $\mu_{m n}(T)=\frac{\pi^{2}}{4}\left((2 m+1)^{2}+(2 n+1)^{2}\right)$, where $m, n=0,1,2, \ldots$ Thus, the first eigenvalue in $T_{1}$ is $\mu_{1}\left(T_{1}\right)=\frac{\pi^{2}}{2 b^{2}}=\frac{4}{(2 \sqrt{2}-1)^{2}} \pi^{2} \geq \pi^{2}$ and the corresponding eigenfunction is $v_{1}(x)=\sin \left(\frac{\pi}{2 b} x_{1}\right) \sin \left(\frac{\pi}{2 b} x_{2}\right)$. Also the first eigenvalue $\mu_{1}\left(T_{2}\right) \geq \pi^{2}$ as observed above. Moreover, $\mu_{1}\left(P_{1,2}\left(\frac{\pi}{4}\right)\right)>2 \pi^{2}$. Therefore in each prism

$$
\frac{\left\|\nabla u ; L^{2}\left(P_{i}\left(\frac{\pi}{4}\right)\right)\right\|^{2}}{\left\|u ; L^{2}\left(P_{i}\left(\frac{\pi}{4}\right)\right)\right\|^{2}} \geq 2 \pi^{2}, \quad \text { where } i=1,2 .
$$

For any $u \in H_{0}^{1}\left(\mathbf{Y}_{R}^{\frac{\pi}{4}}\right)$ having one orthogonality condition $\int_{Q} u(x) v_{1}(x) \mathrm{d} y \mathrm{~d} z=0$, where $v_{1}$ is the first eigenfunction of the mixed boundary value problem on the cuboid $Q$, the inequality

$$
\left\|\nabla u ; L^{2}(Q)\right\|^{2}>2 \pi^{2}\left\|u ; L^{2}(Q)\right\|^{2}
$$

is valid. The Friedrichs inequality for the cylinders is given in (12). Summing up these inequalities ((12), (19) and (20)), we obtain

$$
\left\|\nabla u ; L^{2}\left(\mathbf{Y}_{R}^{\frac{\pi}{4}}\right)\right\|^{2} \geq 2 \pi^{2}\left\|u ; L^{2}\left(\mathbf{Y}_{R}^{\frac{\pi}{4}}\right)\right\|^{2} \quad \text { with one orthogonality condition. }
$$

The assumption of having at least two eigenvalues in the discrete spectrum fails by comparing the inequality (21) and the max-min principle (8).

In addition to the above result, we are able to improve the lemma slightly. 


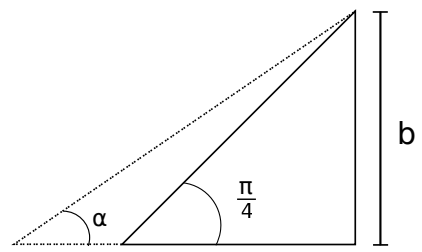

Figure 8. The isosceles right triangle $T_{1}\left(\frac{\pi}{4}\right)$ and the stretched $T_{1}(\alpha)$.

Lemma 5. Let $\alpha \in\left[\arctan \left(\frac{3}{4}\right), \arccos (2-\sqrt{2})\right]$. Then the number of eigenvalues in the discrete spectrum $\sigma^{d}\left(\mathbf{Y}_{R}^{\alpha}\right)$ equals one.

Proof. We follow the paper [14] and perform new (local) variables by setting $\left(y_{2}, z\right) \mapsto\left(y_{2}, z b \cot (\alpha)\right)$. Thus, the triangle $T_{1}\left(\frac{\pi}{4}\right)$ is transformed to $T_{1}(\alpha)$. By the max-min principle,

$$
\begin{aligned}
\mu_{1}\left(\frac{\pi}{4}\right) & =\frac{\pi^{2}}{2 b^{2}}=\min _{u \in H_{0}^{1}\left(T_{1}\left(\frac{\pi}{4}\right)\right)} \frac{\left\|\nabla u ; L^{2}\left(T_{1}\left(\frac{\pi}{4}\right)\right)\right\|^{2}}{\left\|u: L^{2}\left(T_{1}\left(\frac{\pi}{4}\right)\right)\right\|^{2}} \\
& =\min _{v \in H_{0}^{1}\left(T_{1}(\alpha)\right)} \frac{b^{2}(\cot (\alpha))^{2}\left\|\partial_{z} v ; L^{2}\left(T_{1}(\alpha)\right)\right\|^{2}+\left\|\partial_{y_{2}} v ; L^{2}\left(T_{1}(\alpha)\right)\right\|^{2}}{\left\|v: L^{2}\left(T_{1}(\alpha)\right)\right\|^{2}} \\
& \leq \max \left\{(b \cot (\alpha))^{2}, 1\right\} \min _{v \in H_{0}^{1}\left(T_{1}(\alpha)\right)} \frac{\left\|\nabla v ; L^{2}\left(T_{1}(\alpha)\right)\right\|^{2}}{\left\|v: L^{2}\left(T_{1}(\alpha)\right)\right\|^{2}} \\
& =\max \left\{(b \cot (\alpha))^{2}, 1\right\} \mu_{1}(\alpha) .
\end{aligned}
$$

Here $H_{0}^{1}(T)$ is the Sobolev space with smoothness index one having all the functions $u$ which vanish on one leg of the triangle. Thus, $\mu_{1}(\alpha) \geq \min \left\{\left(b^{-1} \tan (\alpha)\right)^{2}, 1\right\} \mu_{1}\left(\frac{\pi}{4}\right)$. Taking into account the restriction (18), we have $\mu_{1}(\alpha) \geq \frac{\pi^{2}}{2 b^{2}}$. Hence, the requirement that the first eigenvalue $\mu_{1}(\alpha) \geq \pi^{2}$ is valid when $\alpha \leq \arccos (2-\sqrt{2})$. Thus on the prisms, the following inequality is valid:

$$
\frac{\left\|\nabla u ; L^{2}\left(P_{i}(\alpha)\right)\right\|^{2}}{\left\|u ; L^{2}\left(P_{i}(\alpha)\right)\right\|^{2}} \geq 2 \pi^{2}, \quad \text { where } i=1,2 .
$$

Summing up the inequalities (12), (20) and (22), leads to

$$
\frac{\left\|\nabla u ; L^{2}\left(\mathbf{Y}_{R}^{\alpha}\right)\right\|^{2}}{\left\|u ; L^{2}\left(\mathbf{Y}_{R}^{\alpha}\right)\right\|^{2}} \geq 2 \pi^{2},
$$

where the function $u$ satisfies one orthogonality condition on the cuboid $Q$. Comparison between the above inequality and the max-min principle (8), yields to the desired result for the three-dimensional domain $\mathbf{Y}_{R}^{\alpha}$.

5.1. The monotonicity of the eigenvalues. We notice that while the angle is varying the smallest area in the center of the waveguide is attained when $\alpha=\frac{\pi}{3}$, that is, an equilateral triangle. For any other angle, the center is greater and has a form of an isosceles triangle. Based on the comparison principle, we will show the following. 

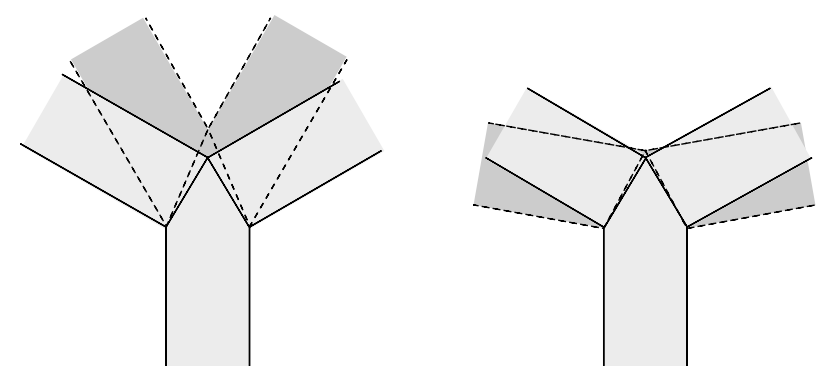

Figure 9. The branching waveguides $\mathbf{Y}^{\alpha}$ and $\mathbf{Y}^{\frac{\pi}{3}}$, where $\alpha \leq \frac{\pi}{3}$.

Lemma 6. The function $\alpha \mapsto \lambda_{p}^{\alpha}$ is strictly increasing when $0<\alpha \leq \frac{\pi}{3}$ and strictly decreasing when $\frac{\pi}{3}<\alpha \leq \frac{\pi}{2}$.

Proof. Let $0<\alpha<\beta \leq \frac{\pi}{3}$ and make a division of the waveguides $\mathbf{Y}^{\alpha}$ and $\mathbf{Y}^{\beta}$ into three cylinders, where the arms form the cylinders $\Pi^{\alpha}, \Pi^{\beta}$ with slanted ends and the legs are the cylinders $\Xi^{\alpha}, \Xi^{\beta}$, respectively, with pointed end, see Fig. 9 and 10.

The cylinder $\Pi^{\alpha}$ is mapped to $\Pi^{\beta}$ by the local transformation, namely, compression $(y, z) \mapsto\left(y, \frac{\tan (\hat{\alpha})}{\tan (\hat{\beta})}\right)$. Here $\hat{\alpha}$ and $\hat{\beta}$ indicates the angles in the slanted ends of the cylinders $\Pi^{\alpha}$ and $\Pi^{\beta}$, respectively, with a property $\hat{\alpha}<\hat{\beta}$. Similarly, to the cylinder $\Xi^{\alpha}$ with the pointed end, we make the local coordinate change, where the cylinder is compressed to $\Xi^{\beta}$. That is, $(y, z) \mapsto\left(y, \frac{\tan (\tilde{\alpha})}{\tan (\tilde{\beta})}\right)$. Here $\tilde{\alpha}$ and $\tilde{\beta}$ denote the angles in the pointed end of the cylinders $\Xi^{\alpha}$ and $\Xi^{\beta}$, and clearly $\tilde{\alpha}<\tilde{\beta}$. Due to the compressions along the cylinders, we obtain via the max-min principle

$$
\begin{aligned}
\lambda_{p}^{\alpha} & =\max _{\mathcal{E}_{p}^{\alpha}} \inf _{u \in \mathcal{E}_{p}^{\alpha} \backslash\{0\}} \frac{\left\|\nabla u ; L^{2}\left(\mathbf{Y}_{R}^{\alpha}\right)\right\|^{2}}{\left\|u: L^{2}\left(\mathbf{Y}_{R}^{\alpha}\right)\right\|^{2}} \\
& =\max _{\mathcal{E}_{p}^{\beta}} \inf _{v \in \mathcal{E}_{p}^{\beta} \backslash\{0\}} \frac{\left\|\partial_{y_{1}} v ; L^{2}\left(\mathbf{Y}_{R}^{\beta}\right)\right\|^{2}+\left\|\partial_{y_{2}} v ; L^{2}\left(\mathbf{Y}_{R}^{\beta}\right)\right\|^{2}+(1+\epsilon)^{-2}\left\|\partial_{z} v ; L^{2}\left(\mathbf{Y}_{R}^{\beta}\right)\right\|^{2}}{\left\|v: L^{2}\left(\mathbf{Y}_{R}^{\beta}\right)\right\|^{2}} \\
& \leq \max _{\mathcal{E}_{p}^{\beta}} \inf _{v \in \mathcal{E}_{p}^{\beta} \backslash\{0\}} \frac{\left\|\nabla v ; L^{2}\left(\mathbf{Y}_{R}^{\beta}\right)\right\|^{2}}{\left\|v: L^{2}\left(\mathbf{Y}_{R}^{\beta}\right)\right\|^{2}}=\lambda_{p}^{\beta} .
\end{aligned}
$$

Let now $\frac{\pi}{3}<\alpha<\beta \leq \frac{\pi}{2}$. Now the cylinders $\Pi^{\beta}$ and $\Xi^{\beta}$ have sharper ends and therefore we compress cylinders of $\mathbf{Y}^{\beta}$ to cylinders of $\mathbf{Y}^{\alpha}$. We proceed as above, but the roles of $\alpha$ and $\beta$ are changed and therefore we obtain by the max-min principle

$$
\lambda_{p}^{\beta} \leq \lambda_{p}^{\alpha},
$$

which finishes the proof.

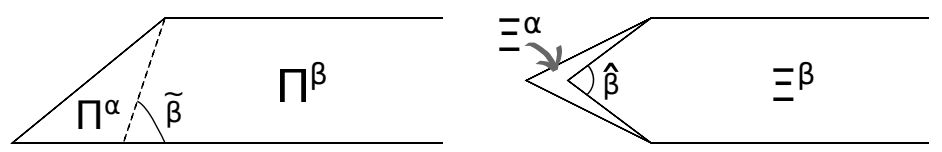

Figure 10. The cylinders $\Pi^{\alpha}, \Pi^{\beta}$ and the pointed end cylinders $\Xi^{\alpha}$ and $\Xi^{\beta}$.

Moreover, when $\alpha=\frac{\pi}{2}$, the Y-shaped waveguide turns into T-shaped waveguide T. This type of planar waveguide was explored in [12]. In the three-dimensional 
case, we divide $\mathbf{T}$ into three cylinders with right-angled end and a cube $\mathcal{C}$ of unit side length located in the junction. For this cube, we pose Neumann condition on the sides $z= \pm \frac{1}{2}$ and $y_{2}=-\frac{1}{2}$. For the other three sides we pose the Dirichlet condition. The first eigenvalue is $\frac{5}{4} \pi^{2}<2 \pi^{2}$ and the corresponding eigenfunction is $v_{1}(x)=\cos \left(\pi y_{1}\right) \sin \left(\frac{\pi}{2}\left(y_{2}-\frac{1}{2}\right)\right)$. We take the subspace of co-dimension one to be

$$
\mathcal{E}_{2}^{\perp}(\mathbf{T})=\left\{u \in H_{0}^{1}(\mathbf{T}): \int_{\mathcal{C}} v_{1}(x) u(x) \mathrm{d} x=0\right\} .
$$

The second eigenvalue is $\frac{9}{4} \pi^{2}$ and is therefore greater than the threshold $2 \pi^{2}$. Thus, for any function $u \in \mathcal{E}_{2}^{\perp}(\mathbf{T})$, the inequality

$$
\frac{\left\|\nabla u ; L^{2}(\mathcal{C})\right\|^{2}}{\left\|u ; L^{2}(\mathcal{C})\right\|^{2}}>2 \pi^{2}
$$

holds. Summing up the above inequality with (12), we obtain

$$
\frac{\left\|\nabla u ; L^{2}(\mathbf{T})\right\|^{2}}{\left\|u ; L^{2}(\mathbf{T})\right\|^{2}}>2 \pi^{2},
$$

where $u \in \mathcal{E}_{2}^{\perp}(\mathbf{T})$. With the help of the max-min principle, it is guaranteed that $\lambda_{2}^{\frac{\pi}{2}}>2 \pi^{2}$ and therefore the discrete spectrum contains only one eigenvalue. Thus, according to the above consideration and Lemma 6 , we derive the following.

Lemma 7. Let $\alpha \in\left[\arctan \left(\frac{3}{4}\right), \frac{\pi}{2}\right]$. Then the number of the eigenvalues in the discrete spectrum $\sigma^{d}\left(\mathbf{Y}_{R}^{\alpha}\right)$ is one.

On the other hand, for the cranked two-dimensional waveguide, Nazarov and Shanin found a critical threshold $\alpha_{*} \approx 0.077 \pi$ so that for any smaller $\alpha$ the multiplicity of the discrete spectrum exceeds one (see [14]). Thus, for any $\alpha<\alpha_{*}$, the second eigenvalue $\lambda_{2}^{\alpha}(V)<\pi^{2}$. Taking into account the third dimension and the domain monotonicity $\mathbf{V} \subset \mathbf{Y}_{R}^{\alpha}$, the number of eigenvalues in the discrete spectrum exceeds one at least for the same threshold $\alpha_{*}$.

5.2. The circular waveguide while the angle $\alpha$ is varying. For the circular waveguide $\mathbf{Y}_{C}^{\alpha}$, where $\alpha$ is varying, the division as in the rectangular case (see Fig. 7) is incompetent since the threshold of the continuous spectrum is $4 j_{0,1}^{2}$ and there is no such $\alpha$ that the second eigenvalue of the cuboid $Q$ would be greater than this threshold. Likewise, we are not able to show the uniqueness of the eigenvalue in the discrete spectrum in the case when $\alpha=\frac{\pi}{2}$, since $\frac{9}{4} \pi^{2}<4 j_{0,1}^{2}$.

We adapt the condition given in [10] for the first positive eigenvalue of the Neumann-Laplacian on the triangle. That is, $\mu_{2}>\frac{j_{1,1}^{2}}{D^{2}}$, where $D$ is the diameter of the triangle and $j_{1,1} \approx 3.8317$ is the first positive root of the Bessel function of order 1 . We split the waveguide like was done in the regular case, that is, into three right-angled cylinder and prism in the middle.

When $\alpha<\frac{\pi}{3}$, we extend the waveguide from the middle by setting a prism with an isosceles triangle as a cross-section (see Fig. 11a)). The extension is needed to attain the right-angled ends to the cylinders. The diameter of the triangle is $D=\frac{\cos (\alpha)(2-\cos (\alpha))}{(\sin (\alpha))^{2}}$. Hence, for the prism, $\mu_{2}>\frac{j_{1,1}^{2}}{D^{2}}+\pi^{2}>4 j_{0,1}^{2}$ when $\alpha>0.326 \pi$. 


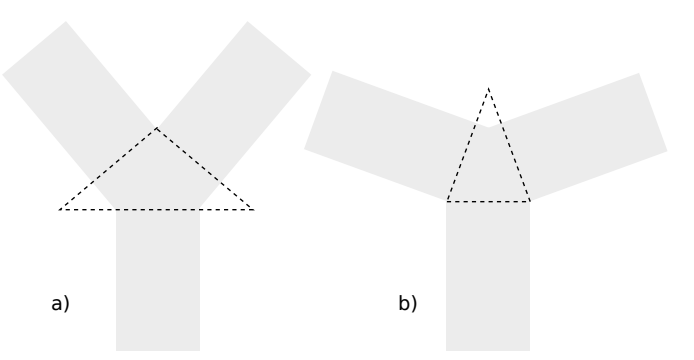

Figure 11. The branching waveguide $\mathbf{Y}_{C}^{\alpha}$ and the extension.

Respectively, when $\alpha \geq \frac{\pi}{3}$, we extend the waveguide by an isosceles triangle with acute vertex (see Fig. 11b)). Now the diameter of the triangle is $D=\frac{1}{2 \cos (\alpha)}$. Thus, $\mu_{2}>\frac{j_{1,1}^{2}}{D^{2}}+\pi^{2}>4 j_{0,1}^{2}$ when $\alpha<0.342 \pi$. The resembling inequality as given in (17) is valid when $\alpha \in(0.326 \pi, 0.342 \pi)$ and hence by the max-min principle, the number of eigenvalues in the discrete spectrum is one whenever $\alpha$ belongs to this small interval around the value $\frac{\pi}{3}$.

We write $\mathbf{Y}_{R(s)}$, where $s$ is the width of the cylinder. The circular waveguide can be situated between two rectangular waveguides, namely, $\mathbf{Y}_{R\left(\frac{1}{\sqrt{2}}\right)} \subset \mathbf{Y}_{C} \subset \mathbf{Y}_{R(1)}$ (see Fig. 12). Certainly, the structure of the spectrum for the $\mathbf{Y}_{R\left(\frac{1}{\sqrt{2}}\right)}$ is similar than for the $\mathbf{Y}_{R(1)}$ with the exception that the threshold for the continuous spectrum is $4 \pi^{2}$. It would be tempting to say that being between these two waveguides the spectrum for $\mathbf{Y}_{C}$ must have the same conditions. However, this may not be true since the threshold of the continuous spectrum is different in each case.

For example, let us assume that for a fixed value of $\alpha$, the multiplicity of the discrete spectrum is one for the rectangular waveguides. That is, $\lambda_{2}\left(\mathbf{Y}_{R(1)}^{\alpha}\right)>2 \pi^{2}$ and $\lambda_{2}\left(\mathbf{Y}_{R\left(\frac{1}{\sqrt{2}}\right)}^{\alpha}\right)>4 \pi^{2}$. Due to the domain monotonicity, it holds that $\lambda_{2}\left(\mathbf{Y}_{R(1)}^{\alpha}\right) \leq$ $\lambda_{2}\left(\mathbf{Y}_{C}^{\alpha}\right) \leq \lambda_{2}\left(\mathbf{Y}_{R\left(\frac{1}{\sqrt{2}}\right)}^{\alpha}\right)$. We note that the eigenvalue $\lambda_{2}\left(\mathbf{Y}_{C}^{\alpha}\right)>2 \pi^{2}$ but we are not able to say whether the eigenvalue is below the threshold $4 j_{0,1}^{2}$ of the continuous spectrum or not.

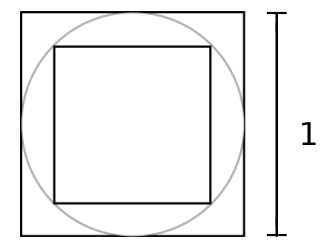

Figure 12. The cross-section of the waveguides $\mathbf{Y}_{R}$ and $\mathbf{Y}_{C}$.

\section{References}

[1] Avishai, Y., D. Bessis, B. G. Giraud, and G. Mantica: Quantum bound states in open geometries. - Phys. Rev. B 44:15, 1991, 8028-8034.

[2] Bandaru, P.: Electrical characterization of carbon nanotube Y-junctions: a foundation for new nanoelectronics. - J. of Materials Sci. 42:5, 2007, 1809-1818.

[3] Birman, M.Sh., and M.Z. Solomyak: Spectral theory of selfadjoint operators in Hilbert space. - D. Reidel Publishing Co., Dordrecht, 1987.

[4] Cardone, G., T. Durante, and S. A. Nazarov: The localization effect for eigenfunctions of the mixed boundary value problem in a thin cylinder with distorted ends. - SIAM J. Math. Anal. 42:6, 2010, 2581-2609. 
[5] Courant, R., and D. Hilbert: Methods of mathematical physics 2: Partial differential functions. - Interscience Publishers, New York, 1953.

[6] Duclos, P., and P. Exner: Curvature-induced bound states in quantum waveguides in two or three dimensions. - Rev. Math. Phys. 7:1, 1995, 73-102.

[7] Exner, P., and P. Šeba: Bound states in curved quantum waveguides. - J. Math. Phys. 30:11, $1989,2574-2580$.

[8] Goldstone, J., and R. L. Jaffe: Bound states in twisting tubes. - Phys. Rev. B 45:24, 1992, 14100-14107.

[9] Lamé, M. G.: Leçons sur la théorie mathématique de l'élasticité des corps solides. - Deuxième édition, Gauthier-Villars, 1866.

[10] Laugesen, R. S., and B. A. Siudeja: Minimizing Neumann fundamental tones of triangles: an optimal Poincaré iequality. - J. Differential Equations, 249, 118-135 (2010).

[11] Morse, P. M., and H. Feshbach: Methods of theoretical physics. Part 1. - McGraw-Hill Science, New York, 1953.

[12] Nazarov, S. A.: Trapped modes in a T-shaped waveguide. - Acoustic Journal 56:6, 2010, 747-758; English transl.: Acoustical Physics 56:6, 2010, 1004-1015.

[13] Nazarov, S. A.: Discrete spectrum of cranked, branching and periodic waveguides. - Algebra i analiz 23:2, 2011, 206-247; English transl.: St. Petersburg Math J. 23:2, 2011.

[14] Nazarov, S. A., and A. V. Shanin: Trapped modes in angular joints of 2D waveguides. Appl. Anal., 2013.

[15] Nazarov, S. A., K. Ruotsalainen, and P. Uusitalo: The Y-junction of quantum waveguides. - Z. Angew. Math. Mech. 94:6, 2014, 477-486.

[16] PAlm, T., and L. ThYLÉN: Designing logic functions using an electron waveguide Y-branch switch. - J. of Applied Physics 79:10, 1996, 8076-8081.

Received 28 May $2014 \bullet$ Accepted 5 September 2014 